\title{
Optimisation Framework for Development of Cost-effective Monitoring in Distribution Networks
}

DOI:

10.1049/iet-gtd.2015.0757

\section{Document Version}

Accepted author manuscript

Link to publication record in Manchester Research Explorer

\section{Citation for published version (APA):}

Liao, H., Liu, Z., \& Milanovic, J. V. (2016). Optimisation Framework for Development of Cost-effective Monitoring in Distribution Networks. IET Generation, Transmission and Distribution, 10(1), 240-246. https://doi.org/10.1049/ietgtd.2015.0757

\section{Published in:}

IET Generation, Transmission and Distribution

\section{Citing this paper}

Please note that where the full-text provided on Manchester Research Explorer is the Author Accepted Manuscript or Proof version this may differ from the final Published version. If citing, it is advised that you check and use the publisher's definitive version.

\section{General rights}

Copyright and moral rights for the publications made accessible in the Research Explorer are retained by the authors and/or other copyright owners and it is a condition of accessing publications that users recognise and abide by the legal requirements associated with these rights.

\section{Takedown policy}

If you believe that this document breaches copyright please refer to the University of Manchester's Takedown Procedures [http://man.ac.uk/04Y6Bo] or contact uml.scholarlycommunications@manchester.ac.uk providing relevant details, so we can investigate your claim.

\section{OPEN ACCESS}




\title{
Optimisation Framework for Development of Cost-effective Monitoring in Distribution Networks
}

\author{
Huilian Liao ${ }^{1}$, Zhixuan Liu ${ }^{1}$, Jovica V. Milanović ${ }^{{ }^{*}}$, Nick C. Woolley ${ }^{2}$ \\ ${ }^{1}$ School of Electrical and Electronic Engineering, The University of Manchester, Manchester, \\ M60 1QD, UK \\ ${ }^{2}$ National Grid, London, UK \\ *milanovic@ manchester.ac.uk
}

\begin{abstract}
This paper presents a novel optimisation methodology, Optimal Placement of Monitors $\left(\right.$ OPM $\left._{\text {Power }}\right)$, for optimal device/monitor placement in distribution networks. $O$ PM $_{\text {Power }}$ is developed based on gradient search and Particle Swarm Optimisation (PSO). The proposed method integrates network topology into search process via spanning trees and uses the historical experience for search guidance. The method is particularly suited for optimal placement problems in power systems. The application is illustrated on the problem of optimal monitor placement for estimation of voltage unbalance in a section of existing UK distribution network and in a generic distribution network. It is demonstrated that the proposed methodology outperforms generic integer optimisation algorithms which are widely used for optimal placement problems in literature, e.g. Genetic Algorithms (GA).
\end{abstract}

\section{List of Abbreviations and Symbols}

OPM $_{\text {Power }} \quad$ Optimal Placement of Monitors

PSO Particle Swarm Optimisation

GA Genetic Algorithms

DNO Distribution Network Operator

VUF Voltage Unbalance Factor

DSSE Distribution System State Estimation

PDF probability distribution function

FEs number of function evaluations

$Z_{i} \quad$ position vector of particle $i$

$z_{i d} \quad$ the $d^{\text {th }}$ dimensional position of particle $i$

$D \quad$ number of monitors to be installed

A branch-to-bus connection matrix

$a_{j k} \quad$ entry corresponding to the $j^{\text {th }}$ row and $k^{\text {th }}$ column of $\boldsymbol{A}$

$Z_{\text {node }} \quad$ current search node

$C\left(z_{\text {node }}\right) \quad$ temporal performance of $z_{\text {node }}$ 
S

$V_{i}$

$P_{i}, p$-best

$P_{g}, g$-best

$N_{l}$

$P S$

$L$

$G\left(z_{i d}\right)$

$c_{1}$ and $c_{2}$

$r_{1}$ and $r_{2}$

w

$N_{\max 1}$

$N_{\max 2}$

VUFA

VUFE

F

$\boldsymbol{R}$

Y

H

X

$U F$ node score

velocity of particle $i$

the best position found by one particle so far

the fittest particle found so far in the swarm

number of nodes on path $l$

path score

total number of paths in the search tree

graphic location of node $z_{i d}$

acceleration factors

random numbers within range $[0,1]$

inertia weight

interior maximum numbers of iterations

exterior maximum numbers of iterations

actual VUF

estimated VUF

objective function

covariance matrix of measurement errors

vector of measurements

a nonlinear set of state equations

state variables

unbalance factor

\section{Introduction}

Optimal monitor/device placement in power networks has been attracting significant attention from both utilities and customers over the years. It is likely to continue to do so considering the importance of network monitoring for development of sustainable and flexible networks of the future. Over the last decade, optimal monitor placement has been extensively considered as the way to efficiently and optimally estimate different phenomena and aspects of power systems performance. The studies include reliable estimation of voltage sags [1, 2], harmonics source estimation [3], general power system state estimation [4, 5], load estimation [6] and assessment of the dynamic performance of the power system [7] etc. This problem, considered as an integer programming problem, can be solved using heuristic search, simulated 
annealing or tabu search, etc. In the past, Genetic Algorithms (GA) are widely applied to solve this placement problem $[2,5,8-10]$. GAs, however, typically suffer from slow convergence and being trapped in local optimum, due to the lack of using historical search experience and practical information of the search environment for search guidance. As optimal placement problems are getting more attention in power system studies in general, it is necessary to develop optimisation algorithm which converges fast and reaches the global optimum accurately. For general optimisation algorithm, the values of variables/inputs are typically used to extract the search gradient information which will be later on used for search guidance. In the case of monitor/device placement problems, the numerical indices of the monitored buses are taken as the inputs. Buses in power systems, however, are interconnected by complex network and bus indices could be, and often are, named arbitrarily, i.e., two close bus indices could represent two buses which are geographically far away from each other, depending on the way of numbering them. If the gradient information is extracted directly from bus indices, it can be meaningless if it is used for search guidance, and the search could be misled due to the inappropriate gradient information. Instead of using a general optimisation algorithm, this study focuses on development of an algorithm which is particularly tailored for optimal placement problems under the search environment of power systems. In this study network topology is used to extract gradient information for search guidance, rather than using the gradient information extracted from bus indices. Topology of power networks has been studied in the past using graph theory [11]. Spanning trees, especially, are particularly useful in observability analysis of power system networks $[12,13]$ and have been applied to improve topological observability $[14,15]$.

In this paper, the problem of monitor placement is illustrated through the estimation of voltage unbalance. The voltage unbalance is chosen as case study due to the increasing attention that it has been receiving from both utilities and customers faced by growing penetration of single phase distributed energy resources (generation and storage). The unbalance leads to thermal stress/aging of power system equipment and user-connected devices, and the additional power/energy loss in the network. These phenomena reduce the efficiency of load and overall network, and consequently result in substantial financial loss to Distribution Network Operators (DNOs) and customers [16]. To limit the potentially harmful consequences of voltage unbalance and apply appropriate mitigating solutions it is necessary to monitor/estimate the unbalance throughout the network in the most cost-efficient way. For voltage unbalance, a number of standards have been developed to specify the performance requirement and evaluation techniques, e.g. EN 50160 [17] and IEC 61000-4-30 [18]. These standards have been used around the world as the basis for development of national quality of supply regulations. Violation of 
thresholds specified in standards could potentially result in heavy penalties imposed to DNOs. To avoid this, proper monitoring and accurate estimation of the unbalance performance are required. In the process of planning and optimal (most cost-effective) monitor placement, both, requirements specified in standards and the uncertainties of measurements and variation of monitoring data should be taken into account [18]. Proper design of a planning framework for unbalance estimation which addresses the aforementioned factors and is able to generate optimal monitor set that is cost-effective in the long run is still required.

This paper proposes a novel optimisation methodology, Optimal Placement of Monitors (OPM Power), based on spanning trees, gradient search and PSO, for optimal monitor placement problems in power systems and illustrates it on a problem of optimal monitor placement for estimation of unbalance in meshed and radial power networks. PSO is chosen due to its simplicity of implementation, few parameters needed and high convergence rate [19]. Differently from the past applications of spanning trees, spanning trees are used here to construct search paths. Node/path scores, used for search guidance, are developed here for the first time to facilitate the integration of network topologies into optimisation search, and thus realize network topology-based search. The paper also introduces a planning framework for optimal monitor placement for unbalance estimation by incorporating voltage unbalance standards in final decision making regarding the number of monitors needed. The accuracy and efficiency of OPM $\mathrm{Power}_{\text {are }}$ demonstrated by comparison with a widely used Genetic Algorithm based optimisation.

\section{Methodology and Framework}

\subsection{Optimisation Methodology}

To find the optimum value (minimum or maximum) of a given function efficiently and accurately, it is important to consider three key aspects: 1) keep a balance between exploration and exploitation; 2) use the search gradient information properly; 3) know the search environment, i.e. the solution space. These aspects have been extensively addressed in the past for continuous numerical optimisation algorithm studies. They, however, are still not paid sufficient attention in the study of optimal placement problems. A dedicated method is therefore proposed in this paper for optimal monitor placement in power systems based on spanning trees, gradient search and PSO.

2.1.1 Initialisation of Monitor Placement: Two particles are employed to search the optimal monitor placement in the study [19]. The position vector of each particle, i.e. the indices of buses at which the monitors will be installed, is denoted as $Z_{i}=\left(z_{i 1}, z_{i 2}, \ldots, z_{i d}, \ldots, z_{i D}\right)$, where $z_{i d}$ represents the $d$ dimensional position of particle $i$, and $D$ represents the number of monitors to be installed in the network. 
The particles are initialised based on network branch-to-bus connection matrix, denoted as $\boldsymbol{A}$, with an entry $a_{j k}$ determined according to the following rule:

$$
a_{j k}= \begin{cases}0 & \text { if branch } j \text { is not connected to bus } k \\ 1 & \text { if branch } j \text { is connected to bus } k\end{cases}
$$

The sum of each column of $\boldsymbol{A}$, representing the number of connections belonging to the corresponding bus, is obtained and used for ranking the buses. Among all buses, the first $D$ buses which have the largest number of connections are selected to form the first particle, as these buses usually provide dispersed search paths. Searching starting from this particle is able to make the best of the search environment, and to improve the efficiency of search.

For the second particle, its initialisation process is the same as that of the first particle, except for the network branch-to-bus connection matrix is built according to a different rule: $a_{j k}=0$ if branch $j$ is not connected to bus $k$, or if branch $j$ is only connected to bus $k$ in the downstream branch; otherwise, $a_{j k}=1$. It can be seen that the network connection matrix is built without considering the branches which are connected to only one single bus in the downstream branch, as it is believed that these branches contribute less to the main network connection topology. The initialisation of the second particle is to select the buses which have more important connections.

2.1.2 Gradient Search: Before performing search, a spanning/search tree is constructed for each bus based on the network topology. For illustration, a section of existing UK meshed distribution network is provided in Fig. 1 (a), and the search tree constructed for bus 14 is given in Fig. 1 (b). This search tree shows that there are 10 possible search paths beginning from the root node. For each dimensionality of particle $Z_{i}$, a gradient search is performed on the search tree that roots from the current dimensional position, i.e. $z_{i d}$. The search starts from root node, and progresses towards the nodes which are connected to the root node. When the search reaches one node (denoted as $z_{\text {node }}$ ), this node will be used to replace the current dimensional position $z_{i d}$. In this way, a new solution $Z_{i}^{\prime}=\left(z_{i 1}, \ldots, z_{i d-1}, z_{\text {node }}, z_{i d+1} \ldots, z_{i D}\right)$ is formed. The performance of node $z_{\text {node }}$, denoted by $C$, is evaluated by:

$$
C\left(z_{\text {node }}\right)= \begin{cases}1 & \text { if } F\left(Z_{i}^{\prime}\right) \leq F\left(Z_{i}\right) \\ 1 & \text { if } z_{\text {node }} \in\left\{z_{i 1}, \ldots, z_{i d-1}, z_{i d+1} \ldots, z_{i D}\right\} \\ 0 & \text { otherwise }\end{cases}
$$




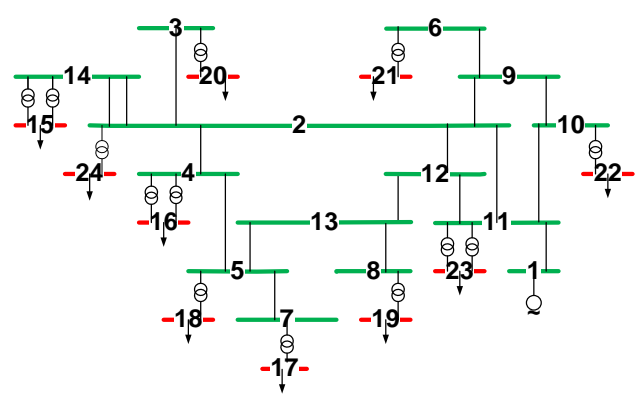

$a$

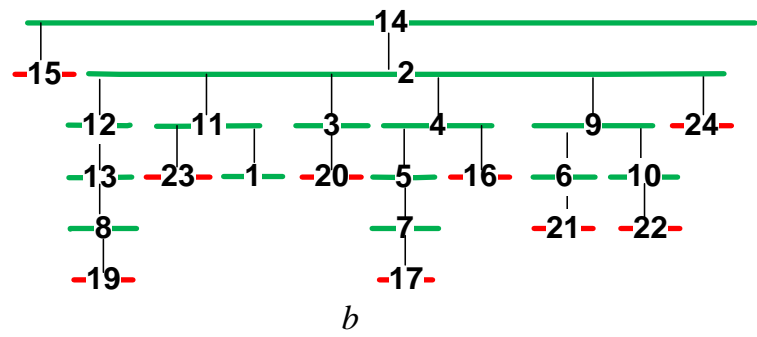

Fig. 1. Illustration of the construction of search tree for bus 14 a 24-bus distribution network

b Search tree for bus 14

In the following gradient search, whether the search proceeds to the nodes that are connected to $z_{\text {node }}$ is determined by $C$. If $C\left(z_{\text {node }}\right)=1$, the search proceeds along the downstream branches of $z_{\text {node }}$, and keeps evaluating the nodes which are connected to $z_{\text {node }}$; otherwise, the search terminates at node $Z_{\text {node }}$. In other words, if node $Z_{\text {node }}$ improves the fitness value compared with the original monitor set, or if the node is one of other dimensionalities in the particle, the search proceeds along the downstream braches; otherwise the search terminates. This is to ensure that the search is focused on potential paths, and to some extent improve the efficiency of search via exploitation. After the gradient search is completed for one dimensionality of the particle, and before gradient search moves to the next dimensionality, the best node found so far for current dimensionality is used to replace the corresponding dimensional position of the particle.

Each node of the tree is associated with a score, which is updated based on $C$. If a node is visited during the gradient search, the score of the node, denoted as $S$, is updated:

$$
S\left(z_{\text {node }}\right)=S\left(z_{\text {node }}\right)+C\left(z_{\text {node }}\right)
$$

It can be seen that if the search reaches one node, and still proceeds to the downstream braches of this node, the score of the node is increased by one. After all dimensionalities perform gradient search in turn, the updated particle positions and the node scores of search trees can be obtained.

2.1.3 Particle Swarm Optimisation (PSO): PSO works on the social behaviour of particles in a swarm by remembering the best location of itself and the best experience of other individuals in the swarm. The particles alter their velocities, denoted as $V_{i}=\left(v_{i 1}, \ldots, v_{i D}\right)$, at each iteration. The best position of particle $i$ ( $p$-best) and the fittest particle found so far in the swarm ( $g$-best) are represented by $P_{i}=\left(p_{i 1}, \ldots, p_{i D}\right)$ and $P_{g}=\left(p_{g 1}, \ldots, p_{g D}\right)$ respectively. Different from general PSO, each iteration of OPM $\mathrm{Power}_{\text {consists of }}$ 
two steps, path selection and searching along the selected paths. This facilitates for the first time integration of network topology into optimisation search.

Step 1 For each dimensionality of the particle, a path to be searched is selected based on probabilities derived from the node scores. For the $d^{\text {th }}$ dimensional position of particle $Z_{i}$, its search path is selected from the search tree which roots from $z_{i d}$. Assume there are $L$ paths in the corresponding search tree, and there are $N_{l}$ nodes, denoted as $\bar{z}_{l, j}, j \in\left\{1, \ldots, N_{l}\right\}$, on path $l, l \in\{1, \ldots, L\}$. The total score of path $l$, denoted as $P S$, is equal to the accumulated node scores along the path:

$$
P S(\text { path } l)=\sum_{j=1}^{N_{l}} S\left(\bar{z}_{l, j}\right)
$$

Given the path scores of $L$ paths, the search path is selected among $L$ paths according to the following probability distribution: if $z_{i d}=p_{i d}$ and $z_{i d} \neq p_{g d}$, the path which node $p_{g d}$ locates on is selected; if $z_{i d} \neq p_{i d}$ and $z_{i d}=p_{g d}$, the path with $p_{i d}$ is selected; if $z_{i d} \neq p_{i d}$ and $z_{i d} \neq p_{g d}$, the path with $p_{g d}$ is selected with a higher probability, $P_{1}\left(p_{1}>0.5\right)$, compared with the path with $p_{i d},\left(1-P_{1}\right)$. If more than one path meets the aforementioned condition or if $z_{i d}=p_{i d}$ and $z_{i d}=p_{g d}$, the path is selected based on $P S$. In this case, the path which has the highest $P S$ is selected with a higher probability, $P_{2}\left(P_{2}>\right.$ $0.5)$, while other paths share the uniform distribution of $\left(1-P_{2}\right) . P_{1}$ and $P_{2}$, selected based on experience, are set to 0.7 and 0.8 respectively in the study, which is to enable more detailed search on potential paths associated with $p_{g d}$ or high $P S$. This procedure of path selection utilizes the gradient information derived previously, and achieves a proper trade-off between exploration and exploitation. The path selection, incorporated with spanning/search trees, is the key procedure to realize the network topology-based optimisation.

Step $2 D$ paths are selected for a particle, while each is corresponding to one dimensionality of the particle. Then the particle will search along these paths simultaneously by altering the particle velocity and graphic position according to:

$$
\begin{gathered}
v_{i d}^{k+1}=w v_{i d}^{k}+c_{1} r_{1}\left(G\left(p_{i d}\right)-G\left(z_{i d}^{k}\right)\right)+c_{2} r_{2}\left(G\left(p_{g d}\right)-G\left(z_{i d}^{k}\right)\right) \\
G\left(z_{i d}^{k+1}\right)=G\left(z_{i d}^{k}\right)+v_{i d}^{k+1}
\end{gathered}
$$

where $k$ is the iteration index, $w$ denotes the inertia weight, $r_{1}$ and $r_{2}$ denote random numbers within range [0,1], constants $c_{1}$ and $c_{2}$ are acceleration factors, which allow particles to adjust its trajectory based on $p$ best and $g$-best, $G\left(z_{i d}\right)$ refers to the graphic location of node $z_{i d}$ on the path, while the velocity $v_{i d}$ represents the number of nodes the particle flies over on the selected path. During the process of optimisation, the dimensional positions of the particle are updated simultaneously, in order to avoid the particle being trapped in local optima. Once the graphic locations of dimensional positions are updated 
using (6), the particle position will be replaced with the corresponding nodes. At the end of each iteration, $P_{i}$ and $P_{g}$ are updated with the $p$-best and $g$-best solutions found so far respectively.

2.1.4 Optimisation Process: The process of optimisation which includes search tree, gradient search and PSO is given in Fig. 2, where $N_{\max 1}$ and $N_{\max 2}$ represent the interior and exterior maximum numbers of iterations respectively.

Fig. 2. Flow chart of $O P M_{\text {Power }}$

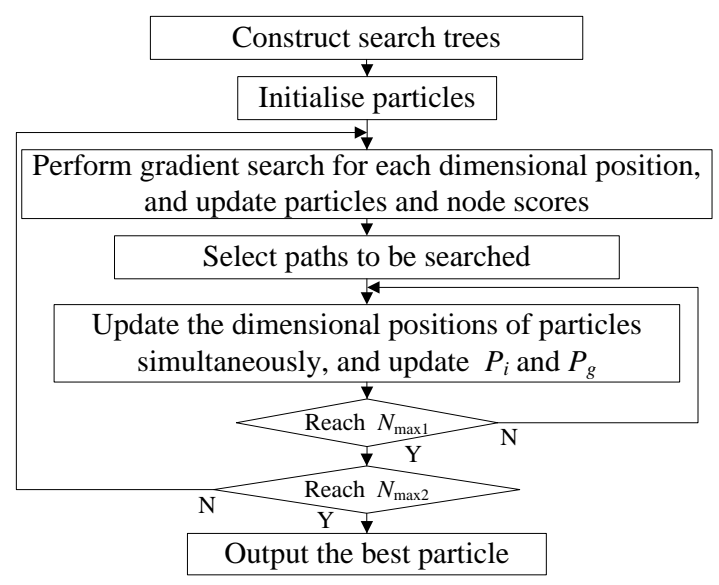

\subsection{Problem Definition}

To assess the unbalance severity of an individual bus, Voltage Unbalance Factor (VUF), defined as the ratio of negative to positive sequence voltage, is applied to present the unbalance severity of a bus in the study [18]. The problem of optimal monitor placement is defined as a minimisation problem, i.e., to reduce the error between the true unbalance profile and that estimated based on the monitoring data. Given a set of monitors, $Z$, the objective function (denoted as $F$ ) can be defined as:

$$
F(Z)=\sum_{i=1}^{N_{\text {bus }}}\left|\operatorname{VUFE}_{i}(Z)-V U F A_{i}\right|
$$

where $V U F A_{i}$ denotes the actual VUF of bus $i, V U F E_{i}(Z)$ represents the VUF estimated based on monitor set $Z, N_{\text {bus }}$ represents the total number of buses in the network. In (7), absolute error rather than relative error is used. If relative error is used, the buses with high VUFs will have less influence on the total estimation error $F(Z)$, compared to the buses with small VUFs. This can be derived from the denominator of the relative error formula, $\frac{\left|V U F E_{i}(\boldsymbol{Z})-V U F A_{i}\right|}{V U F A_{i}}$. Accurate estimation of buses with high VUFs, however, is more important than estimation of buses with small VUFs from the perspective of compliance with voltage unbalance standard.

To estimate the unbalance profiles of buses based on monitoring data, state estimation is applied in the study. The state estimation problem is defined as: 


$$
\min _{X}[Y-H(X)]^{T} \boldsymbol{R}^{-1}[Y-H(X)]
$$

where $Y$ denotes a vector of measurements, $H(X)$ represents a nonlinear set of equations that describes the true state of the power system with state variable $X$, and $\boldsymbol{R}$ is the covariance matrix of measurement errors $E, E=Y-H(X)$, which is a vector of errors between the observed measurements and true state of the system. In the study, Distribution System State Estimation (DSSE) method, incorporated with pseudomeasurements is adopted for unbalance estimation [20].

In the study, the uncertainties of pseudo-measurements and monitoring data are taken into account by adding normally distributed errors to the measured and monitored data. To account for the uncertainties, Monte Carlo simulations are used in conjunction with DSSE. One-hundred Monte Carlo scenarios were generated by running the state estimator with 100 sets of measurements, which are distributed as defined by the error properties of the pseudo-measurements and real monitoring measurement. Thus $100 V U F E_{i}$ are obtained for each bus. With the set of $V U F E_{i}$, probability distribution function (PDF) is developed for each bus based on the distribution of $V U F E_{i}$. Normalized distribution functions are obtained for the estimated VUF in the study, as normalized distributed measurement errors are applied. With the constructed PDFs, the most probable value is taken as the final estimated VUF of bus $i$, and it will be employed to calculate the estimation error $F$ in (7).

\subsection{Flow Chart of the Framework}

The overall framework for optimal monitor placement for voltage unbalance assessment is given in Fig. 3. The level of unbalance of the unbalanced load is presented by an unbalance factor (UF). One unbalance estimation/evaluation considering uncertainty can be described by the following two steps: 1) given the power network and load profiles, the uncertainty of unbalanced load is accounted for by adding normally distributed variation $\pm 15 \%$ with 3 SIGMA to $U F$, and then load flow analysis is performed to obtain $V U F A_{i}$;2) given a set of monitors, the uncertainty of pseudo-measurements and variance of monitoring data are included in the evaluation by adding normally distributed errors to the measured and monitored data (refer to [20] for the ranges of the errors imposed to various measurements), and then DSSE is fed with the monitored data in order to calculate $V U F E_{i}$. With $V U F A_{i}$ and $V U F E_{i}$, the fitness value (i.e., estimation error) of the given set of monitors can be evaluated using (7). With this estimation/evaluation available, the proposed optimisation methodology is applied to optimise the location of the monitors by minimising the estimation error, i.e. the error between the true unbalance profile and the profile estimated by DSSE. In the framework, the range of number of monitors to be installed is specified at the beginning. For each proposed number of monitors, an estimation error is calculated. After 
all considered numbers of monitors are applied individually, the relationship curve between the estimation errors and the number of monitors used is drawn.

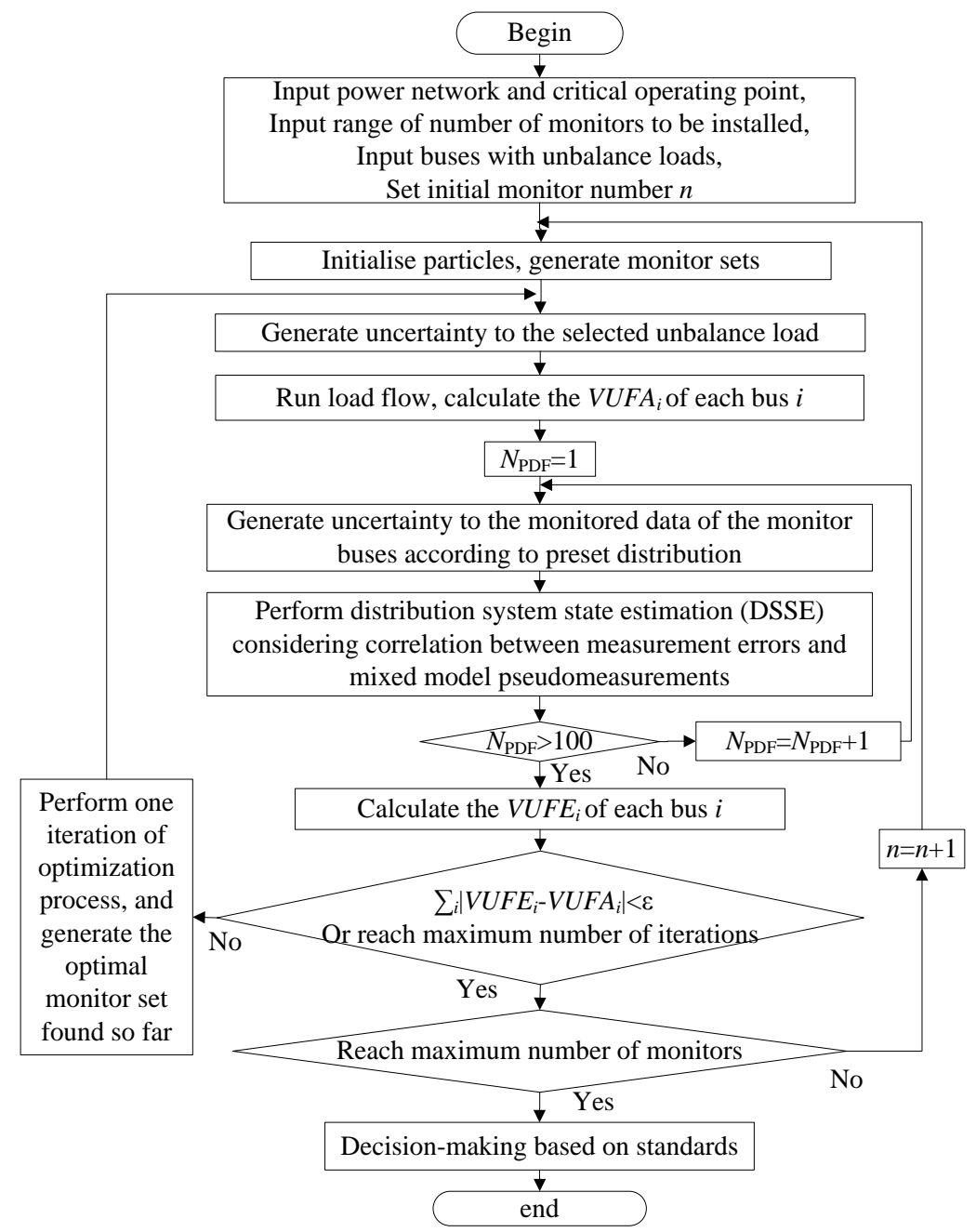

Fig. 3. Framework of monitor placement for unbalance estimation

Due to the significant influence of standards on power quality regulations, the recommended levels of unbalance performance and evaluation requirements specified by standards are taken into account in the final step of the framework, i.e., decision-making. The required number of monitors is determined based on standards, and it is increased only when the violation of standards cannot be identified, or the unbalance performance of the buses that are at the edge of violating the standards cannot be estimated accurately. This is further discussed and illustrated in Section 3. The inclusion of standard specified threshold enables DNOs to assess which buses are violating regulatory limits (so the equipment connected at these buses may overheat or ultimately get disconnected) and such avoid the penalties imposed to DNOs and reduce the financial loss to both DNOs and customers caused by equipment trips/overheating. In the study, EN 
50160 and IEC 61000-4-30, which are widely accepted by regulators, are taken as references. In EN 50160, the required level of voltage unbalance factor is up to $2 \%$ for $95 \%$ of week in low and medium voltage distribution systems. IEC 61000-4-30 specifies that minimum measurement interval should be one-week, and the worst-case values might be compared to contractual values. Similar to EN 50160, IEC 61000-4-30 recommends that one or more $95 \%$ probability weekly values might be used for comparison. Based on these specifications, VUF obtained from $95 \%$ of the worst week is used to compare to the required level of $2 \%$ in the study. With the given required level of unbalance performance, the turning point (knee point) of the relationship curve is assessed by applying corresponding optimal monitor set in the network and simulating network operation for one week. If this optimal monitor set is able to identify the critical buses (i.e. the buses which violate the standard and the buses which are at the edge of violating the standards), the proposed number of monitors is justified and the corresponding monitor set is selected as final solution; if not, the number of proposed monitors is increased, and the next knee point of the relationship curve is tested following the same process. This procedure continues until the least number of monitors which are able to identify all critical buses is obtained.

In this framework, the standard specified threshold for unbalance is not incorporated in the objective function directly due to: 1) If the standard is integrated into the objective function, the optimisation methodology would attempt to find the monitor set which can only accurately estimate the unbalance profiles at the buses which violate the standard without considering other buses which are about to violate the standard specified threshold, e.g., the buses whose VUF is slightly less than $2 \%$, or the buses whose VUF is much larger than $2 \%$ for $<5 \%$ of a week. In this case, the obtained monitor set is only suitable for certain operating condition, and could be infeasible in the long run, as the network topology, loading and operation are subject to daily, weekly and seasonal variations. The buses which are on the edge of violating the standard, in particular, can easily become buses that violate the standard with slight change in the network. This issue will be addressed in Section 3.1;2) To assess appropriately the unbalance profile of the whole network, the information of the maximum VUF in the network is required. However, if the standard is applied to construct the objective function and at the same time the bus which has the maximum VUF does not violate the standard, the maximum VUF in the network cannot be accurately estimated.

In the proposed framework, the overall network unbalance profile is used to derive the appropriate objective function to ensure estimation accuracy at buses with high VUFs, as discussed in Section 2.2. The standard requirement is taken into account in the step of decision-making. This approach overcomes the issues discussed above which could arise if the standard is incorporated in the objective function directly. 
The proposed framework is particularly useful and effective for the assessment of unbalance over longer period of time, and provides sufficiently accurate estimate of the unbalance profiles of the whole network.

\section{Simulation Results}

\subsection{4-bus Meshed Network}

3.1.1 Comparison between $O P M_{\text {Power }}$ and GA: OPM $\mathrm{Power}_{\text {is }}$ compared with a widely used GA based optimisation, on a section of existing UK meshed distribution network as shown in Fig. 1(a). For both $\mathrm{OPM}_{\text {Power }}$ and GA, the number of monitors to be installed is first set to $D=3$ and then to $D=4$. The results are given in Table 1. The number of function evaluations (FEs), i.e., times of performing estimation represents, is also provided in Table 1, as FEs is generally used to compare the computation efficiency among different optimisation algorithms. The effectiveness of $\mathrm{OPM}_{\text {Power }}$ can be presented by the fact that

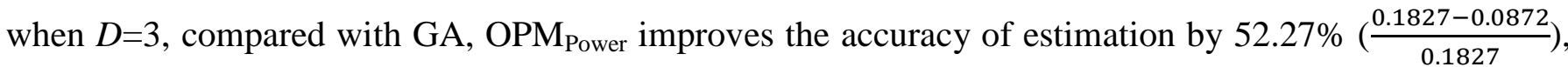
while using $51.25 \%$ less FEs. The results obtained by the two methods when $D=4$ are also given in Table 1 . Compared with the case of $D=3$, the performance of GA degrades when $D=4$, as in this case GA is trapped into local optimum and cannot escape from it. As for $\mathrm{OPM}_{\text {Power, }}$ the performance is improved when one more monitor is installed.

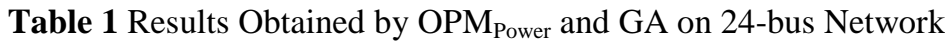

\begin{tabular}{llll}
\hline & Monitors & fitness value $(F)$ & FEs \\
\hline OPM $_{\text {Power }}$ & $3(2,5,11)$ & 0.0872 & 78 \\
GA & $3(2,11,18)$ & 0.1827 & 160 \\
OPM & $4(2,5,9,11)$ & 0.0082 & 110 \\
GA & $4(1,1,2,21)$ & 0.8025 & 260 \\
\hline
\end{tabular}

From the perspective of standards, the inaccuracy of estimation at some buses is acceptable, e.g., in case when both the actual and estimated VUFs at a bus are much smaller/larger than the standard threshold (2\%). However, for those buses whose VUF is around $2 \%$, the accuracy of the estimation is important. To explain this aspect, the VUFs estimated by the two methodologies at buses 19 and 21 and the relative errors are provided in Table 2 . For bus 19 , the actual VUF is slightly larger than $2 \%$. As for bus 21 , the actual VUF is slightly less than $2 \%$, and it is at the edge of violating the standard threshold. It should be mentioned that VUF at bus 21 can easily go beyond $2 \%$ due to future network evolution, which confirms why the standard is not directly integrated into the objective function, as discussed in Section 2.3. In Table 2 , the estimated values which are considered to be accurate enough from the perspective of standard are

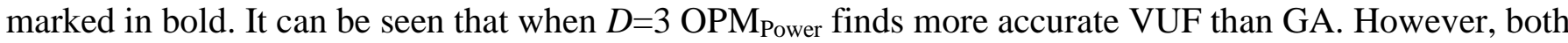


methodologies overestimate the VUF, and fail to identify the unbalance severity regarding the upper limit of standard (2\%) when $D=3$. If this unbalance profile last for $5 \%$ of a week, the assessment based on 3 monitors fails to identify the critical buses regarding the standard. The VUF estimated with four monitors is also given in Table 2. It can be seen that with $D=4$, the VUF estimated by OPMPower is very close to the actual one, and its results are accurate enough to identify the critical buses from the perspective of standard. When $D=4$, VUF estimated by GA at bus 21 is almost the same as the actual value, as a monitor is placed at bus 21 if the solution obtained by GA is adopted. The small difference (i.e., 0.001) between the VUF estimated by GA (1.986) and the actual VUF (1.987) at bus 21 is due to the inclusion of uncertainty in the monitored data. Although GA provides more accurate VUF at bus 21, it fails to identify the critical bus 19 , where standard threshold is violated.

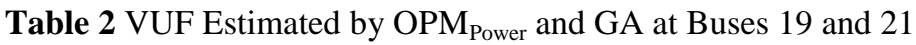

\begin{tabular}{lllll}
\hline & \multicolumn{2}{l}{ VUF [\%] } & \multicolumn{2}{l}{ Relative errors [\%] } \\
\hline & Bus 19 & Bus 21 & Bus 19 & Bus 21 \\
Actual & 2.029 & 1.987 & N/A & N/A \\
OPM $_{\text {Power }}(D=3)$ & $\mathbf{2 . 0 3 2}$ & 2.035 & 0.15 & 2.42 \\
GA $(D=3)$ & $\mathbf{2 . 0 8 3}$ & 2.041 & 2.66 & 2.72 \\
$\operatorname{OPM}_{\text {Power }}(D=4)$ & $\mathbf{2 . 0 2 8}$ & $\mathbf{1 . 9 9 4}$ & 0.05 & 0.35 \\
$\operatorname{GA}(D=4)$ & 1.875 & $\mathbf{1 . 9 8 6}$ & 7.59 & 0.05 \\
\hline
\end{tabular}

3.1.2 Decision Making: Based on the proposed framework, the relationship curve between the number of

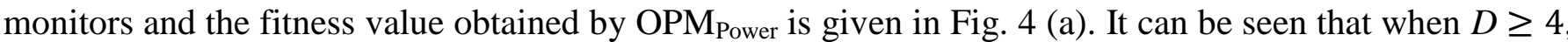
the obtained fitness value converges to a very small value. Even when the number of monitors is increased to a value larger than 4 , the improvement of assessment accuracy is negligible compared to that when $D=4$. Based on Fig. 4 (a), the solution with $D=4$ is tested by simulation of weekly operation of the network. The monitor set $\left(2,5,9\right.$ and 11) obtained by $\mathrm{OPM}_{\text {Power }}$ is simulated in this case. The load profiles of all loads in the network (in total 10 loads) are based on real on-site measurements, taken during the week in August 2012 in UK. Various types of loads are included in the network, and each load has its specific load profile, representing a certain type of customers, i.e., residential, commercial and industrial. The profile of the total load demand in the network, i.e. the sum of the load demands of the ten loads, is presented in Fig. 4 (b). In total, there are 1008 operating points each representing the operation during a ten minute period. The unbalance is generated by unevenly distributing the load demand among three phases at the representative loads located at buses 15, 17, 19 and 21 respectively [20]. The load distribution among the three phases is guided by, i.e., it is made proportional to, the total load demand at the buses using UF. The obtained three phase demands are used to perform load flow study and $V U F A_{i}$ is calculated for each bus. The $V U F A_{i}$ of 
the monitored buses, i.e., the monitored data coming from the monitor set, is fed into DSSE, and $V U F E_{i}$ of the non monitored buses is estimated. From the perspective of standard requirement, only bus 15 violates the standard specified threshold. The monitor set obtained by OPM $_{\text {Power }}$ accurately identifies the critical bus (i.e. bus 15). For illustration, the true and estimated VUFs obtained at the four important buses (i.e. buses 15, 17, 19 and 21) are given in Fig. 5. The 1008 VUFs are sorted in ascending order, and 5\% of the highest VUFs within a week are separated using a green dash-dotted line. It can be seen that the actual and estimated VUFs overlap. This demonstrates the accuracy of VUFs estimated at critical buses using the monitor set obtained by $\mathrm{OPM}_{\text {Power }}$.
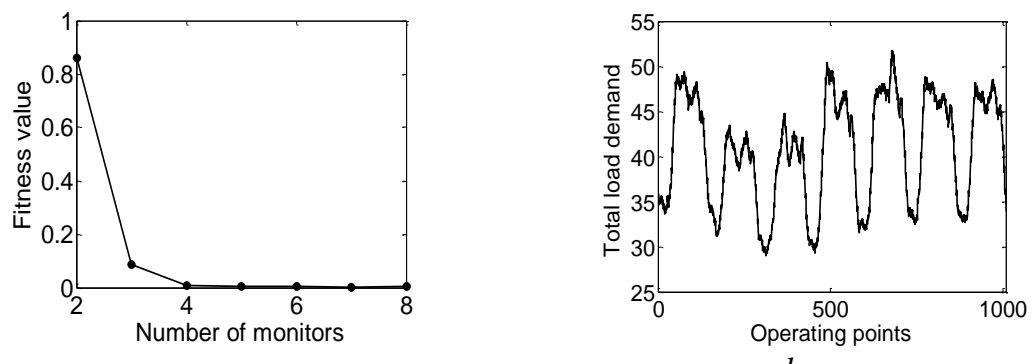

$a$

$b$

Fig. 4. Relationship curve between monitor number and fitness value based on 24-bus network \& the actual load profiles taken a week in August 2012

a Relationship curve

b Actual load profile
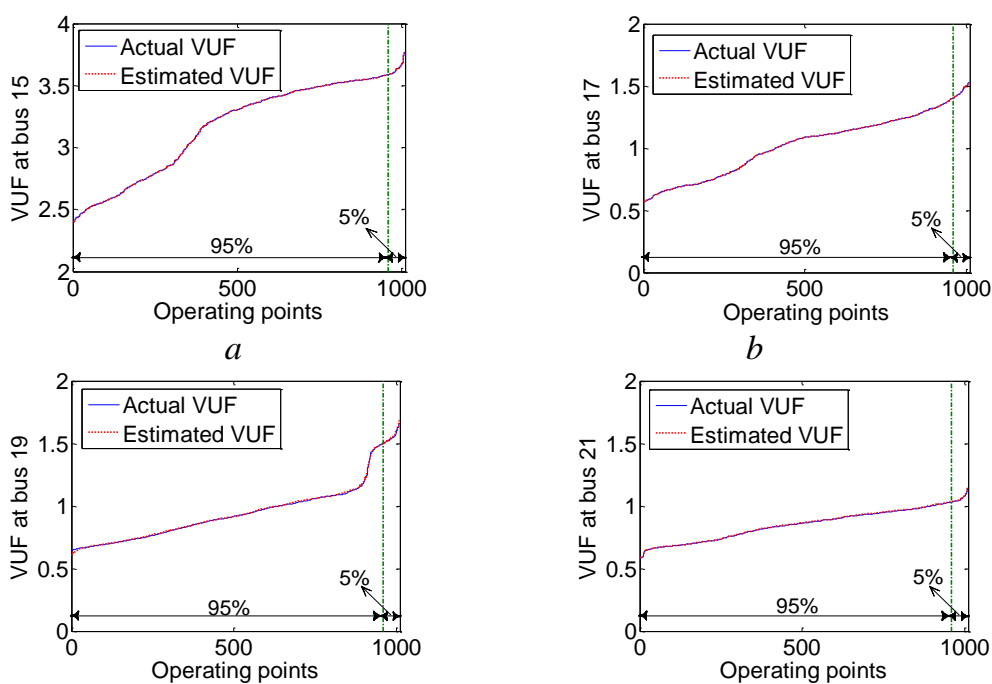

$b$

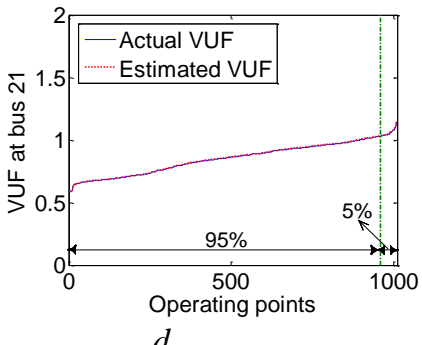

$c$

$d$

Fig. 5. VUF based on 1008 practical operating points within a week

a VUF at bus 15

b VUF at bus 17

c VUF at bus 19

d VUF at bus 21

\subsection{6-bus Radial Distribution Network}

The developed framework is further validated on a 96-bus radial section of a generic UK distribution 
network used in the past studies e.g., [21-24]. The single line diagram of the network is shown in Fig. 6. The number of monitors is set to 4, and the results obtained by OPMPower and GA are given in Table 3. It can be seen that $\mathrm{OPM}_{\text {Power }}$ improves the accuracy of estimation by $35.61 \%$. The heat-map is employed to present the unbalance profiles estimated by $\mathrm{OPM}_{\text {Power, }}$ as shown in Fig. 6. The areas exposed to unbalance, i.e. the three areas circled with the red dashed lines, can be easily identified.

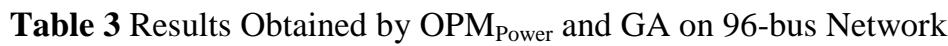

\begin{tabular}{llll}
\hline & Monitors & fitness value $(F)$ & FEs \\
\hline OPM $_{\text {Power }}$ & $4(146,166,174,206)$ & 1.4025 & 260 \\
GA & $4(129,147,193,204)$ & 2.1783 & 260 \\
\hline
\end{tabular}

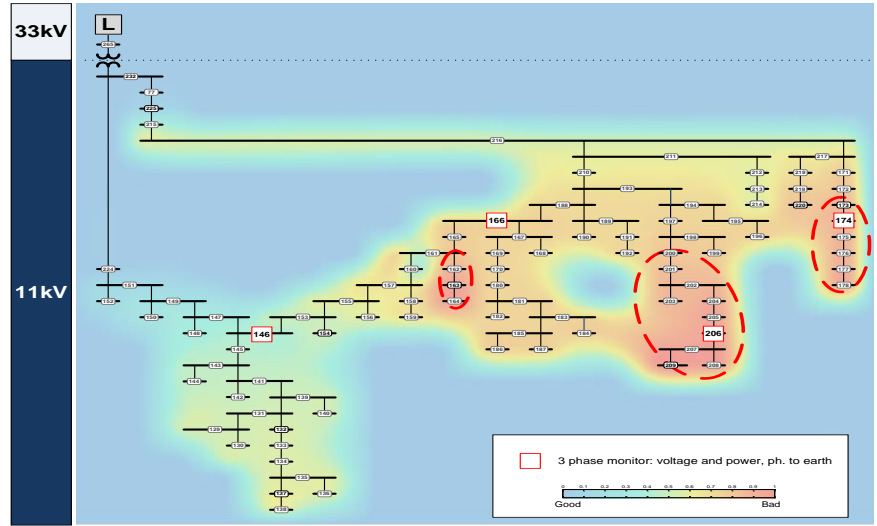

Fig. 6. Heat-map of VUF estimated by OPM Power on 96-bus network with 4 monitors at buses 146, 166, 174 and 206

The relationship curve between the fitness value and monitor number is given in Fig. 7 (a). It can be seen that the estimation becomes more accurate if more monitors (appropriately distributed in the network) are used. Based on the relationship curve, a decision-making process (the last step of the proposed framework) is performed to determine the smallest number of monitors required to identify the critical buses, i.e., the buses which violate standards and those at the edge of violating standard threshold. It can be seen that 4 and 6 monitors determine the decline trend of fitness value as the monitor number increases. The solution with 4 monitors is tested using a week's operation. It was found that among the 96 buses, the VUF at buses 207, 208 and 209 is larger than $2 \%$ for $>1 \%$ of a week. The true and estimated VUF at these three buses is presented in Fig. 7 (b), (c) and (d) respectively, where the standard threshold (2\%) and 5\% of the highest VUF within a week are marked using green dash-dotted lines. The intersection point of the two green dash-dotted lines divides the area of the graph into four segments. If the actual and estimated VUFs cross the same segments of the green dash-dotted lines, the monitor set is able to identify the critical buses with regard to standard requirements. It can be seen from Fig. 7 (b) and (c), that actual and estimated VUFs cross the same segments of the margin lines, i.e. the right and bottom segments of the green dash- 
dotted lines. For Fig. 7 (d), both the actual and estimated VUFs are larger than 2 for $>5 \%$ of a week. It can be seen that the $\mathrm{OPM}_{\text {Power }}$ is able to allocate optimally a relatively small set of monitors, in order to accurately identify the critical buses in the network from the perspective of standard compliance.

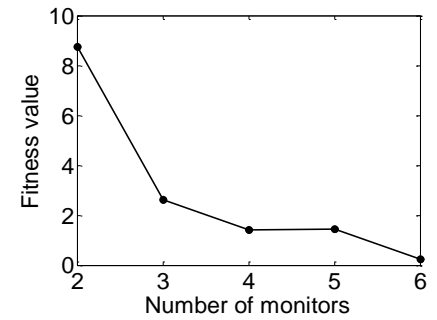

$a$

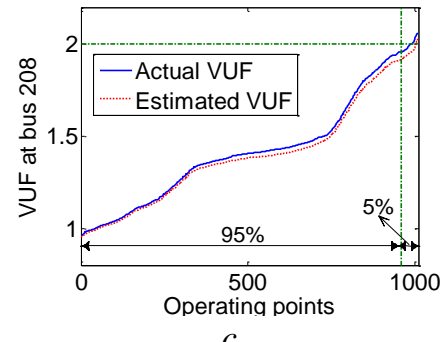

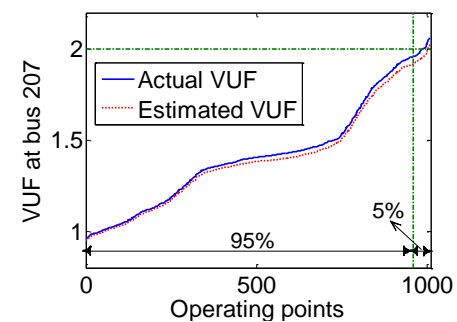

$b$

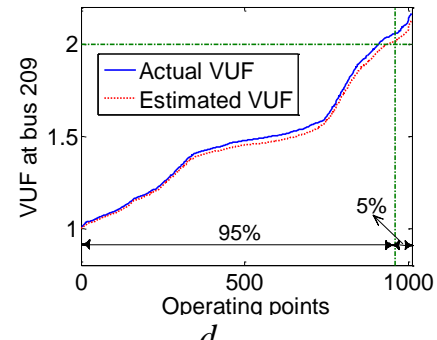

Fig. 7. Relationship curve for 96-bus network and the VUF obtained by a week's operation a Relationship curve

b VUF at bus 207

c VUF at bus 208

d VUF at bus 209

\section{Conclusions}

This paper presented a new methodology $\left(\mathrm{OPM}_{\text {Power }}\right)$ for generic optimal placement problems in the

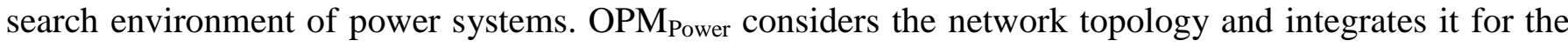
first time into optimisation search via search/spanning trees. By relying on the historical search experience and topology-based search, it improves the overall accuracy and efficiency of the optimisation. Comparison of $\mathrm{OPM}_{\text {Power }}$ with a widely used Genetic Algorithm based optimisation demonstrates its superiority both, in terms of accuracy and efficiency. For a 96-bus realistic test network with four monitors, $\mathrm{OPM}_{\text {Power }}$ improves the accuracy of estimation by $35.61 \%$ compared to GA.

The paper also presents an optimisation based framework for monitor placement for unbalance estimation in distribution networks. It takes into account the uncertainties of pseudo-measurements, variance of monitoring data and standard requirements specified in EN 50160 and IEC 61000-4-30 standards. The simulation results show that the optimal monitor placement derived by OPM $\mathrm{Power}_{\text {, with a }}$ relatively small set of monitors, can successfully identify the critical buses in both meshed and radial distribution networks. 


\section{Acknowledgments}

This work was partly supported by SuSTAINABLE Project under Grant 308755 and partly by Western Power Distribution.

\section{References}

[1] Avendano-Mora, J.M., Milanović, J.V.: 'Monitor placement for reliable estimation of voltage sags in power networks’, IEEE Trans. Power Del. , 2012, 27, (2), pp. 936-944

[2] Kazemi, A., Mohamed, A., Shareef, H., Zayandehroodi, H.: 'Optimal power quality monitor placement using genetic algorithm and Mallow's Cp', Int. Journal of Elect. Power \& Energy Syst., 2013, 53, pp. 564-575

[3] Hartana, R.K., Richards G.G.: 'Harmonic source monitoring and identification using neural networks', IEEE Trans. Power Syst., 1990, 5, (4), pp. 1098-1104

[4] Abbasy, N.H., Ismail, H.M.: 'A unified approach for the optimal PMU location for power system state estimation’, IEEE Trans. Power Syst., 2009, 24, (2), pp. 806-813

[5] Coser, J., Costa, A.S., Rolim J.G.: 'Metering scheme optimization with emphasis on ensuring bad-data processing capability’, IEEE Trans. Power Syst., 2006, 21, (4), pp. 1903-1911

[6] Liu, H., Yu, D., Chiang, H.D.: 'A heuristic meter placement method for load estimation', IEEE Trans. Power Syst., 2002, 17, (3), pp. 913-917

[7] Kamwa, I., Grondin, R.: 'PMU configuration for system dynamic performance measurement in large, multiarea power systems', IEEE Trans. Power Syst., 2002, 17, (2), pp. 385-394

[8] Olguin, G., Vuinovich, F., Bollen, M.H.J.: 'An optimal monitoring program for obtaining Voltage sag system indexes’, IEEE Trans. Power Syst., 2006, 21, (1), pp. 378-384

[9] Souza, J.C., Filho, M.B.D.C., Schilling, M., Capdeville, C.: 'Optimal metering systems for monitoring power networks under multiple topological scenarios’, IEEE Trans. Power Syst., 2005, 20, (4), pp. 1700-1708

[10] Haghifam, M.R., Malik, O.P.: 'Genetic algorithm-based approach for fixed and switchable capacitors placement in distribution systems with uncertainty and time varying loads', IET Gener. Transm. Distrib., 2007, 1, (2), pp. 244-252

[11] Zhu, J.: 'Power systems applications of graph theory' (Nova Science Pub. Inc., 2009)

[12] Mori, H., Tsuzuki, S.: 'A fast method for topological observability analysis using a minimum spanning tree technique', IEEE Trans. Power Syst., 6, (2), pp. 491-500, 1991

[13] Monticelli, A., Wu, F.F.: 'Network observability: theory', IEEE Power Eng. Review, 1985, PER-5, (5), pp. 3233

[14] Mori, H.: 'A GA-based method for optimizing topological observability index in electric power networks'. Proc. IEEE Conf. Evol. Comp., Orlando, FL, 1994, pp. 565-568

[15] Mori, H., Saito, M.: 'A hybrid approach of parallel tabu search and ordinal optimization to meter placement for improving topological observability’. Proc. Int. Conf. Power Syst. Tech., Singapore, 2004, pp. 963-968 
[16] Lglesias, J.G. et al.: ‘Economic framework for power quality’ (JWG CIGRE-CIRED C4.107, 2011)

[17] EN 50160:2004: 'Voltage disturbances standard EN 50160 - voltage characteristics in public distribution systems', 2004

[18] IEC 61000-4-30:2003: 'Testing and measurement techniques - Power quality measurement methods', 2003

[19] Liao, H.L., Ji, Z., Wu, Q.H.: 'A novel genetic particle-pair Optimizer for vector quantization in image coding'. Proc. IEEE Con. Evo Com., Hongkong, 2008, pp. 708-713

[20] Woolley, N.C., Milanović, J.V.: 'Statistical estimation of the source and level of voltage unbalance in distribution networks', IEEE Trans. Power Del., 2012, 27, (3), pp. 1450-1460

[21] Milanović, J.V., Gupta, C.P.: 'Probabilistic assessment of financial losses due to interruption and voltage sags part II: practical implementation', IEEE Trans. Power Del., 2006, 21, (2), pp. 925-932

[22] Zhang, Y., Milanović, J.V.: 'Global voltage sag mitigation with FACTS based devices', IEEE Trans. Power Del., 2010, 25, (4), pp. 2842-2850

[23] Woolley, N.C., Avendano-Mora, J.M., Milanović, J.V.: 'Methodology for robust monitoring of voltage sags based on equipment trip probabilities', Electric Power Syst. Research, 2012, 90, (1), pp. 107-116

[24] Woolley, N.C., Avendano-Mora, J.M., Woolley, A.P., Preece, R., Milanović, J.V., 'Probabilistic estimation of voltage sags using erroneous measurement information', Electric Power Syst. Research, 2014, 106, (1), pp. 142-150 